\title{
Molecular dynamics and spectroscopic insights into iron(III) stabilization of water-soluble polysaccharides
}

\author{
PIOTR ZARZYCKI ${ }^{1}$, AMRITA BHATTACHARYYA ${ }^{1,2}$, \\ CHRISTIAN DEWEY ${ }^{1,3}$, JENNIFER PETT-RIDGE ${ }^{2}$ AND \\ PETER S NICO ${ }^{1}$ \\ ${ }^{1}$ Lawrence Berkeley National Laboratory \\ ${ }^{2}$ Lawrence Livermore National Laboratory \\ ${ }^{3}$ Stanford University \\ Presenting Author: ppzarzycki@lbl.gov
}

Aqueous iron ions and iron (oxyhydr)oxides are known to stabilize soil organic matter (SOM) against decomposition and mineralization in terrestrial ecosystems. However, the nature of the stabilizing interactions remains elusive [1]. Previous studies suggest that SOM is protected either due to Fe crosslinking with organic polymeric molecules or OM co-precipitation with/ or soption to iron (oxyhydr)oxides [1,2,3]. It is also known that the fate of environmental Fe is governed by interactions with organic matter [2].

Here, we combine molecular dynamics (MD) simulations with synchrotron-based spectroscopy and microscopy techniques (STXM/NEXAFS and XAS) to show that the interactions of $\mathrm{Fe}(\mathrm{III})$ with SOM depend on the type and number of reactive functional groups present in SOM. Specifically, we examined $\mathrm{Fe}(\mathrm{III})$ interaction with two water-soluble polysaccharides, amylose (an only hydroxyl containing polysaccharide) and alginate (a carboxyl group containing polysaccharide), because they represent the most abundant class of organic compounds produced in the biosphere.

EXAFS data show that the local Fe-coordination environments in both amylose and alginate complexes are similar. However, molecular dynamics simulations clearly indicate different modes of coordination of $\mathrm{Fe}$ (III) with amylose and alginate. Fe(III) was found to be weakly interacting with amylose forming outersphere complexes. However, the reduction in Fe(III) mobility and increase in its local concentration near the amylose chains was sufficient to trigger co-precipitation of Fe minerals within the amylose matrix. On the other hand, the carboxyl groups in alginate coordinate strongly with $\mathrm{Fe}(\mathrm{III})$, forming inner-sphere complexes. Additionally, we observe an increased rigidity in the alginate matrix, with $90 \%$ of complexed $\mathrm{Fe}(\mathrm{III})$ involved in either crosslinking polysaccharide polymers or bridging carboxylic groups of a single polymer.

We show that amylose and alginate, despite having similar effects on iron local bonding patterns, have entirely different modes of interaction with $\mathrm{Fe}$. Our observations are relevant for understanding how interactions with polyvalent ions stabilize $\mathrm{OM}$ and emphasize how the $\mathrm{OM}$ composition is critical in determining modes of interactions.

[1] M. von Lützow, et al., European Journal of Soil Science 57 (2006) 426-445.

[2] Y.K. Henneberry, et al., Organic Geochemistry 48 (2012) $81-89$.

[3] C. Chen et al. Environ. Sci. Technol. 48 (2014) 13751- 